\title{
El Comercio Justo como \\ alternativa para la redefinición \\ de los vínculos mercantiles a escala local
}

\section{Esteban Cuatrín}

Docente investigador de la Facultad de Ciencias Económicas, Universidad Nacional del Litoral, Argentina.

\section{Lucas Cardozo}

Integrante del Programa de Extensión de Economía Social y Solidaria, Secretaría de Extensión de la Universidad Nacional del Litoral, Argentina. Maestrando en Economía Social, Universidad Nacional General Sarmiento

\section{Eduardo Alfaro}

Docente de la Facultad de Ciencias Económicas, Universidad Nacional del Litoral, Argentina.

\section{Resumen}

Desde las últimas décadas del siglo pasado, el Comercio Justo (CJ) se ha instaurado como un nuevo modo de recreación de las formas tradicionales de comercialización. Si bien el surgimiento de este movimiento estuvo orientado a reformular las relaciones comerciales Norte-Sur, recientemente se ha a abocado a encauzar relaciones Sur-Sur encastradas en distintos valores que materializan dichos principios en formas mercantiles novedosas. Con el desarrollo de la Economía Social y Solidaria (ESS), el CJ aparece como una herramienta para el desarrollo de estas iniciativas orientadas a la construcción de otra economía, en base a valores de solidaridad, cooperación e igualdad. De esta manera, en el artículo presentamos, a partir de algunas reflexiones teóricas e históricas sobre el desarrollo del $\mathrm{CJ}$, una agenda de actividades para su promoción vinculada con una experiencia particular que se desarrolla en el proyecto de extensión que se lleva adelante en la Universidad Nacional del Litoral.

Palabras clave

- Comercio Justo

- economía social

- espacio rural

- construcción social de mercados
Economía Social y Solidaria /

Perspectivas

RECEPCIÓN: 26/06/15

ACEPTACIÓN FINAL: 27/08/15

\section{Resumo}

Desde as últimas décadas do século passado o Comércio Justo (CJ) tem sido estabelecido como um novo modo de recreação das formas tradicionais de comercialização. Embora o surgimento deste movimento estava dirigido a repensar as relações comerciais Norte-Sul, recentemente tentou encaminhar as relações Sul-Sul que monstrasem novas formas de entender os valores e materializar esses princípios em novas formas comerciais. Com o desenvolvimento da Economia Social e Solidária (ESS), o CJ aparece como uma ferramenta para o desenvolvimento destas iniciativas orientadas à construção de outra economia, com base nos valores de solidariedade, cooperação e igualdade. Dessa forma, no artigo apresentamos, a partir de algumas reflexões teóricas e históricas sobre o desenvolvimento do $\mathrm{CJ}$, uma agenda de atividades para sua promoção ligada com uma experiência particular que se desenvolve no projeto de extensão que é realizado pela Universidade Nacional do Litoral.

Palavras-chave

- Comércio Justo

- economia social

- espaço rural

- construção social dos mercados
Para citación de este artículo

Cuatrín, E.; Cardozo, L. y Alfaro, E. (2015). El Comercio Justo como alternativa para la redefinición de los vínculos mercantiles a escala local. En Revista $+E$ versión digital, (5), pp. 104-112. Santa Fe, Argentina: Ediciones UNL. 


\section{Introducción}

Como consecuencia de las reestructuraciones experimentadas en los espacios periurbanos a partir de los años 70 , y fundamentalmente a partir de la década del ' 90 , tanto por la expansión del uso residencial como por el avance de la producción agrícola a gran escala (Craviotti, 2007), muchos actores tradicionalmente vinculados a estos espacios se han visto afectados en sus formas de llevar adelante sus actividades productivas y reproductivas. A su vez, estos actores los pequeños productores- están fuertemente invisibilizados y tienen una escasa posibilidad de interlocución con el Estado en sus distintos niveles. En muchos casos, han dirigido su acción a la constitución de espacios alternativos de producción y comercialización donde se persiguen distintos objetivos de índole político, social, cultural y ambiental (King y Pearce, 2010). Estas iniciativas, articuladas bajo los principios de solidaridad y reciprocidad, se enarbolan dentro del amplio campo de la Economía Social y Solidaria ${ }^{1}$ (ESS),

"en tanto produce sociedad y no sólo utilidades económicas, porque genera valores de uso para satisfacer necesidades de los mismos productores o de sus comunidades — generalmente de base territorial, étnica, social o cultural—y no está orientada por la ganancia y la acumulación de capital sin límites. Además, porque vuelve a unir producción y reproducción, al producir para satisfacer de manera más directa y mejor las necesidades acordadas como legítimas por la misma sociedad" (Coraggio, 2002:286).

Es a partir de las redes conformadas por estos movimientos que acceden a ciertos recursos materiales y simbólicos (Luna García, 2006 y Zibechi, 2010) logran mantenerse y, en algunos casos, inician trayectorias de reproducción ampliada.
Y si bien estas redes han dotado de ciertos recursos a las organizaciones, las posibilidades de registrar procesos de reproducción ampliada, abandonando el puro carácter de actividades de subsistencia, se ven limitadas por las instituciones mercantiles que desde la perspectiva de muchos actores predisponen de un escenario para la desaparición de pequeños productores.

En la configuración antecedente, consideramos al Comercio Justo (CJ) como una iniciativa que bajo distintas formulaciones es apropiable como estrategia para el sostenimiento y fortalecimiento de pequeños productores y otras organizaciones sociales que buscan sostener prácticas tradicionales, valores comunitarios y distintas prácticas ambientales y socialmente sustentables (Lazzaretti Picolotto, 2008 y Coscione, 2013). El CJ se ha propuesto históricamente alterar la configuración institucional de las relaciones mercantiles a las que se les atribuye los procesos empobrecedores o exclusores de pequeños productores. Sus prácticas y valores están basados en la cooperación, solidaridad, reciprocidad, participación, justa distribución de los ingresos producidos, transparencia, entre otros, así como la preocupación respecto de las cualidades intrínsecas del producto que se comercializa y su sustentabilidad ambiental. En este sentido, podemos destacar dos ejes del CJ. Por el lado de la oferta, a partir del fortalecimiento de las organizaciones de pequeños productores y un trabajo de traducción de los principios éticos movilizados por las organizaciones en cualidades de los productos. Por el lado de la demanda, en la reflexión de las actividades de consumo a partir de la concientización y difusión de las pautas del consumo responsable. experiencias de ESS en Argentina, que superaron a las tradicionales o fundantes, como son las cooperativas y mutuales, tales como las denomina Pastore (2010). 
Entendiendo los circuitos mercantiles del CJ como espacios mercantiles relativamente desacoplados de las reglas de los mercados tradicionales pero insertas en los mismos, es necesario avanzar no sólo en el reconocimiento de los valores y el tipo de relaciones del que los productos de CJ son portadores, sino $-\mathrm{y}$ en la medida en que esto no entre contradicción de los principios del CJ-en ciertas dimensiones cualitativas a las que el mercado en su estado actual hace énfasis (seguridad, estandarización, presentación, servicios asociados al producto, etc. ) buscando facilitar el acceso de los integrantes de las organizaciones a tecnologías adaptadas a sus necesidades.

Aunque los principios de $\mathrm{CJ}$, han sido adoptados por muchas organizaciones, la experiencia en el terreno muestra la necesidad de dotar de organicidad y dirección a esfuerzos que muchas veces permanecen aislados. Organizaremos esta presentación en cuatro secciones. En la primera nos abocaremos a un comentario sintético en torno a la situación de emergencia de nuevos espacios de comercialización y producción en el marco de las nuevas ruralidades. A continuación desarrollaremos, desde una perspectiva histórica, la propuesta del CJ para luego, en la tercera sección, proponer una definición de trabajo del CJ. En cuarto lugar proponemos una agenda de trabajo para el desarrollo de la propuesta del CJ con las organizaciones de pequeños productores.

\section{EI CJ como un camino de fortalecimiento del espacio de la ESS en el marco de emergencias de nuevas formas de ruralidad}

Históricamente, el espacio rural era concebido como un sustrato exclusivo para la producción agropecuaria. No obstante, durante las últimas décadas, y sin abandonar esta función, ha ido incorporando diversas actividades ligadas a la necesidad de una redinamización de lo rural ante las transformaciones iniciadas a fines del siglo XX (Nogar, 2008). En determinados casos, los usos del suelo rural pueden alternarse o superponerse, como en el caso del agroturismo que consiste en un tipo de turismo rural vinculado estrechamente con el desarrollo de actividades agropecuarias (Zusman, et al., 2006), por nombrar alguna de estas nuevas funcionalidades.

En medio de estos cambios, el espacio rural se reestructura en su organización y en el uso que le da al territorio (Mikkelsen, 2007). De esta manera comenzó a afianzarse una nueva ruralidad en Argentina, la cual se caracteriza por nuevos vínculos entre los grupos sociales, muchos de ellos pequeños productores que a partir de la construcción de una identidad fuertemente arraigada en el espacio local, y donde la preocupación por las consecuencias sociales y ambientales del modelo productivo dominante llevaron a la adopción de nuevas prácticas (o recuperaron prácticas ancestrales) orientadas por los principios de sustentabilidad, resilencia, y equidad, como es el caso notablemente de la producción agroecológica (Stassart et al., 2012, Altieri y Toledo, 2011, Casadinho, 2013).

En este sentido, en la ESS, y en especial en el CJ, se rescata la identidad de los espacios locales, como ámbitos de construcción de relaciones sociales, sin idealizar estos espacios, sino teniendo en cuenta las formas de dominación que en ellos se despliegan y teniendo en cuenta que en estos espacios permean diferentes escalas que co-construyen las identidades locales.

Por lo tanto, y de acuerdo con lo planteado hasta el momento, el concepto de nueva ruralidad se emplea para expresar la multifuncionalidad de las geografías rurales. Al respecto, el trabajo de Cittadini, et al. (2010) ha visibilizado los modos alternativos de construcción del espacio rural vinculado a la ESS.

Tal como sostiene Van der Ploeg et al. (2012), en los espacios rurales en respuestas a las tendencias concentradoras de la agricultura industrial $-\mathrm{y}$ de otros procesos expulsores conexosse llevan adelante iniciativas de desarrollo rural destinadas tanto a la reducción de la pobreza, a la generación de oportunidades de empleo, el trabajo en pos de la seguridad alimentaria así como un conjunto amplio de objetivos de diversa índole. ${ }^{2}$ Estos procesos de desarrollo rural dan lugar a la emergencia de nuevos mercados, ya sea al crear nuevas conexiones, nuevos canales, o desarrollar nuevas estructuras de gobernanza en los mercados existentes o en nuevos mercados. Estos mercados funcionan con una lógica diferente de los mercados tradicionales, y se caracterizan por su encastramiento social. Si bien todos los mercados están encastrados en determinadas estructuras sociales, los mercados tradicionales están encastrados en estructuras significativamente diferentes de aquellas en las que se encastran los nuevos mercados emergentes. Mientras los mercados tradicionales buscan ofuscar estas estructuras, en el caso de los mercados anidados (según la denominación propuesta por Van Der Ploeg), los productores y consumidores se reconocen y se fortalecen mediante la diferenciación de los productos a raíz de las características sociales y distintivas que le atribuyen a los mismos.
2) Estas contratendencias han sido descritas como un fenómeno general de una reacción autoprotectora de

la sociedad frente al desarrollo de la economía de mercado en el análisis de Karl Polanyi. El autor denomina doble movimiento a este fenómeno (Polanyi, 1989). 


\section{EI CJ como alternativa}

\section{para la transformación de los mercados}

Dentro de estos procesos de formación de espacios mercantiles alternativos, el CJ aparece como un camino de instauración de relaciones económicas novedosas coadyuvantes a la construcción de una ESS (Valero, 2013). El CJ representa una forma de organizar los circuitos mercantiles con vocación de transformación de los mercados tradicionales.

Históricamente comienza a desarrollarse en los años 60 (si bien algunos autores rastrean los primeros antecedentes en el siglo XIX en los intercambios entre Inglaterra y la India colonial). En la primera Conferencia de las Naciones Unidas sobre Comercio y Desarrollo (UNCTAD) varias resoluciones fueron adoptadas con el objetivo de una mayor transferencia de riqueza del norte al sur a la vez que se abogó por una reducción de las políticas proteccionistas de los países del norte bajo la consigna trade but no taid. Aunque estas resoluciones prácticamente no fueron implementadas, la UNCTAD se constituye como un espacio de debate y de usina de las ideas que darán posteriormente lugar a la emergencia de redes de CJ. Estas redes estuvieron en sus orígenes influenciadas por las ideas de los teóricos de la dependencia y las desigualdades inherentes al sistema capitalista mundial. De esta forma procuran la creación de un espacio de intercambio alternativo, que no sea gobernado por las reglas del intercambio internacional sino por actores puestos en pie de igualdad y guiándose por principios de solidaridad (Fridell, 2006). En los años 80 este movimiento y estas organizaciones experimentan una división entre las que adoptan una estrategia de continuidad en la construcción de redes alternativas (lo que se denomina enfoque filière o cadena integrada) y aquellas adoptaron un enfoque centrado en la certificación y que buscaron integrar los productos certificados a las grandes cadenas de distribución en pos de lograr una aumento significativo en el volumen de las ventas de productos provenientes del comercio justo (enfoque certificación). El éxito cuantitativo del segundo enfoque ha llevado a que en muchos casos el CJ se identifique con las reglas y los mecanismos utilizados por las organizaciones que impulsan esta forma de desarrollar el CJ. ${ }^{3}$ Si bien ambos enfoques difieren, tanto en la forma en que se organizan las cadenas de valor, los mecanismos de transmisión de la información e incluso la definición operativa de los principios del CJ, ambas engloban un conjuntos de referenciales en torno al $\mathrm{CJ}$ que presentan grandes similitudes. Los principios del CJ, según la WFTO (Organización Mundial del Comercio Justo), ${ }^{4}$ son los siguientes:

1) creación de oportunidades para los productos en desventaja para combatir la pobreza y lograr un desarrollo sustentable; 2) transparencia y responsabilidad en la administración y en las relaciones comerciales;

3) constitución de capacidades para desarrollar la independencia de los productos;

4) promoción del comercio justo a través de la difusión de información sobre sus prácticas;

5) pago de un precio justo acordado a través del diálogo y la participación, que permite una producción socialmente justa y ambientalmente amigable;
3) Fundamentalmente la Federación de Organizaciones de Certificación de Comercio Justo, FLO (Fairtrade Labelling Organization).
4) Ver: http://comerciojusto.org/ que-es-el-comercio-justo/definicion/ los-10-principios-internacionales/ 


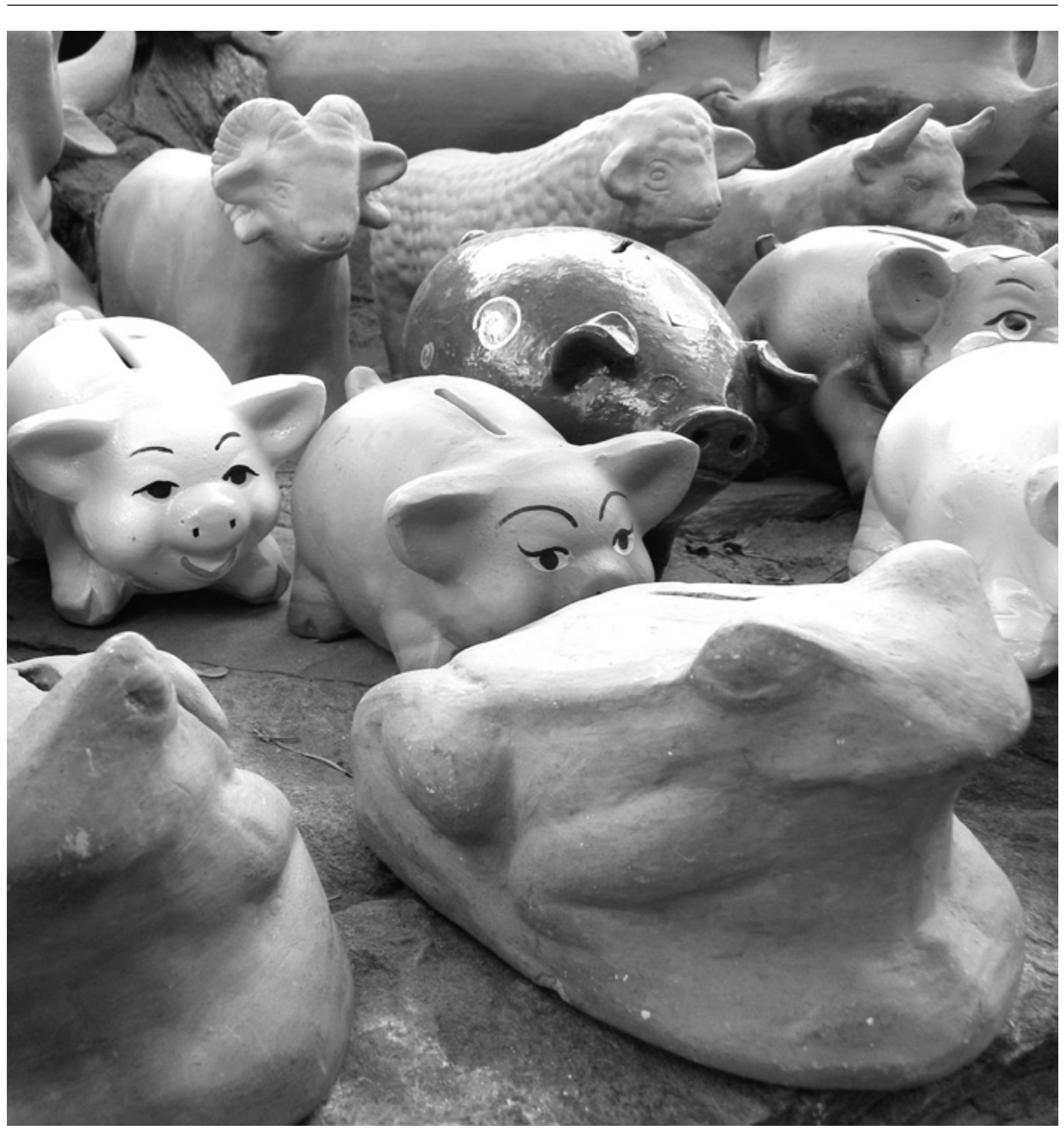


6) equidad de género en la remuneración y en las oportunidades de trabajo;

7) condiciones de trabajo favorables en un ambiente seguro y saludable para los productores;

8) respeto por los derechos de los niños y niñas garantizados por la Convención de la ONU y por leyes y normas sociales locales; 9) conservación del medio ambiente por medio de prácticas ambientales y utilización de métodos de producción responsables y 10) relaciones de comercio basadas en el interés por el bienestar social, económico y ambiental de los pequeños productores, en un marco de solidaridad, confianza y respeto mutuo.

Junto a esta forma histórica de CJ, denominado en la literatura comercio "Norte-Sur" podemos identificar las corrientes "NorteNorte" o "Sur-Sur". En el primer caso se desarrolla a partir de una mayor conciencia en torno a las dificultades de los pequeños productores locales y a la luz de las teorías del desarrollo territorial y local (Le Velly, 2011). En el segundo caso, ha cobrado mayor interés en las últimas décadas y refleja la voluntad de un desarrollo autónomo basado en la articulación de distintas iniciativas de la ESS: "innumerables iniciativas económicas asociativas y cooperativas, redes socioproductivas de comercio ético, comercio comunitario y comercio solidario" (Gomes, 2007:101).

\section{Una definición provisoria del $\mathrm{CJ}$}

Si bien no hemos encontrado una definición amplia de CJ, que integre ambas vertientes históricas (Sur-Sur, y Norte-Sur), del análisis de las mismas y partiendo de una visión donde la variedad de arreglos institucionales posibles dan lugar a distintas formas de funcionamiento de los mercados, ${ }^{5}$ podemos proponer los siguientes elementos de definición de CJ como base para nuestro trabajo:
- En tanto mercado anidado, implica una estructura particular de mecanismos de gobernanza, donde los pequeños productores tienen una mayor capacidad de definir las reglas que estructuran los intercambios (Van der Ploeg et al., 2012) y donde priman las reglas de decisión democráticas y la horizontalidad en las relaciones entre los actores.

- Un tipo distinto de actores tiene un lugar central, incluyendo tanto pequeños productores familiares como distintos actores vinculados al movimiento de la ESS.

- Si bien la estrategia del CJ parte de la construcción de canales alternativos, tiene vocación de transformación de las reglas del comercio y para ello las organizaciones de $\mathrm{CJ}$ asignan una gran relevancia a las actividades de difusión y de concientización de los consumidores aumentando la reflexividad en las decisiones de consumo. ${ }^{6}$

- La producción y comercialización bajo una serie de principios éticos que incluyen la internalización en la evaluación mercantil de los aspectos laborales, ambientales, sociales, culturales identitarios, de cooperación, autogestión y asociatividad, de igualdad de género en los que se lleva adelante la producción. Esta perspectiva "integral" del CJ lo distingue de otras formas de capitalismo ético tales como las iniciativas vinculadas a la Responsabilidad Social Empresaria. Si bien en torno a un conjunto de valores pueden definirse distintos principios operacionales, algunas veces fuertemente controversiales -como es el caso del precio justo-, lo que caracteriza al CJ es la búsqueda de explicitar estos principios.

- Dado que el CJ se construye sobre el reconocimiento de atributos de tipo credence, ${ }^{7}$ implica una forma de gobernanza de la calidad al interior de los mercados donde prime la transparencia en las transacciones y la generación de mecanismos que instituyan la confianza en el seguimiento de los principios éticos que sustentan.
5) Entendiendo los mercados como construcciones sociales históricamente situadas. Si bien el espacio de este marco teórico no permite desarrollar las perspectivas en las que nos basamos algunas obras de referencia de la sociología económica y del institucionalismo económico fundamentales se pueden consultar en Swedberg (2005) y Coriat y Weinstein (2004).
6) Esta labor se debe llevar a cabo teniendo en cuenta que las distintas cadenas de valor, y los sistemas agroalimentarios en particular, están abrumadoramente controladas por poderosos actores e intereses, corporizados en grandes transformadores y fundamentalmente en la gran distribución. Tener altos estándares éticos no es suficiente para posicionarse en un mercado de productos éticos. Es necesario tener soporte y reconocimiento de la sociedad civil e incluso -en algunos casos- que medien acciones de clase, por lo que se deben forjar distintas formas de solidaridad entre las mismas (Lang, 2010).
7) Hace referencia a un tipo de atributos de la calidad de los bienes, que a diferencia de los atributos de búsqueda y experiencia — según la clásica distinción de Nelson (1970)-, no se puede evaluar a partir del consumo de los mismos. 
Con este fin distintos tipos de diseño institucional pueden ponerse en funcionamiento: certificación de tercera parte, sistemas participativos de garantías, o mecanismos centrados en la confianza interpersonal.

\section{Agenda para el fortalecimiento del $\mathbf{C J}$ en la escala local} Atento a la perspectiva histórico-conceptual del movimiento del CJ, así como de la situación concreta de las organizaciones con que nos encontramos interactuando, hemos planteado la siguiente agenda que consideramos puede servir de referencia para otros proyectos que busquen impulsar la transformación de relaciones mercantiles existentes. Esta agenda está planteada pensando en el trabajo conjunto con organizaciones de pequeños productores rurales o rururbanos que, como tratamos de reflejar precedentemente, consideramos — con sus prácticas contestatarias de las formas productivas y de comercialización dominante- constituyen el actor clave en la construcción de estos nuevos espacios.

El primero de los temas es la promoción, el reconocimiento y la adopción de los principios de CJ como una estrategia de fortalecimiento de las mismas. Para ello se deben difundir los principios del $\mathrm{CJ}$ entre las organizaciones de pequeños productores participantes, recoger los valores promovidos por las organizaciones, que tal como se sostiene en Rodet (2015) muchas veces se ubican en la interfase de organización económica y movimiento social. Esta actividad debe conducir a la construcción de una identidad colectiva que tiene un doble objetivo. Por un lado, favorecer la movilización de sus miembros, poder dar un sentido común a la acción del colectivo y poder entablar relaciones con otras organizaciones que persiguen fines similares, a la vez que se fortalece la visibilidad de la organización en el espacio público. La construcción de una identidad colectiva fuerte es parte de la acción protestataria de los movimientos sociales: constituye un recurso para la movilización de los miembros y para su visibilidad en el espacio público (Neveu, 1996, citado en Rodet, 2015). Estas identidades deberán traducirse en algún tipo de protocolo o carta de principios generales que guíen la producción y comercialización y que traduzcan en términos concretos los principios perseguidos por el colectivo. El tipo de dispositivo adoptado dependerá del grado de organización y de los recursos materiales disponibles por las organizaciones y los productores. La experiencia muestra que definiciones estrictas y dispositivos complejos como las certificaciones pueden resultar impracticables en la realidad local y una carta de principios, basada en algunos referenciales "flexibles" puede ser la herramienta más asequible para la mayoría de las organizaciones de pequeños productores familiares, al menos en un primer momento.

En este sentido, el grupo de extensión puede contribuir a la definición de innovaciones sociales mercantiles a partir de la identificación y recopilación de protocolos y de esquemas de comercialización que puedan servir como modelos.

Otro de los temas es la creación de canales o mercados que respondan a otras lógicas, diferentes a las imperantes en los mercados capitalistas, hay que tener en cuenta que esta disociación se enmarca en el funcionamiento de los mismos, con lo cual los mercados deben responder en cierta medida a los imperativos del mercado siempre y cuando esto no entre en contradicción con los principios éticos promovidos. De esta forma aparece la necesidad de trabajar en términos de estandarización de los productos así como en las formas de presentación y la regularidad en la provisión de los mismos.

Las organizaciones de pequeños productores adolecen frecuentemente de la falta de servicios de apoyo disponibles por otros tipo de actores productivos, por ese motivo es de fundamental importancia coadyuvar a la perdurabilidad de las organizaciones a partir del fortalecimiento jurídico, contable, organizativo y comunicacional de las mismas. El grupo de extensión puede tener un rol importante aquí en la creación de nuevos vínculos entre la organización y otras organizaciones y organismos que puedan acompañar y/o financiar proyectos que apunten a la reforma de las prácticas productivas y de comercialización.

La otra gran tarea en la promoción del CJ pasa por la necesidad de "proyectar hacia afuera, de visibilizar la existencia de otra cultura de producción y consumo de carácter responsable y 
superar a medio plazo la barrera de los ya convencidos hasta llegar a un público general menos concienciado" (Arnold y Galán, 2014:112).

Teniendo en cuenta la gran variedad de medios de difusión, y la necesidad de apoyarse en redes, prácticas y recursos preexistentes, nos parece importante destacar dos aspectos a trabajar en este punto: contribuir a la visibilización de las organizaciones y favorecer un mayor reconocimiento del $\mathrm{CJ}$ y de sus principios.

En este punto es importante dar reconocimiento a las organizaciones que suelen estar fuertemente invisibilizadas. Para ello se contempla la producción de materiales (a ser distribuidos en distintas actividades de difusión) que permitan el reconocimiento de la situación social, su ubicación territorial, el tipo de problemáticas que enfrentan. Para ello algunas herramientas como las cartografías sociales o participativas pueden ser de gran utilidad (IFAD, 2009 y Tetamanti y Escudero, 2012), en el sentido de que permiten identificar distintos aspectos y problemáticas sociales que emergen del territorio y a partir de la mirada de los actores

El otro conjunto de actividades están vinculadas al reconocimiento no solo de los principios del CJ, sino también (y fundamentalmente) a la toma de conciencia de la naturaleza política del acto de consumo. Las experiencias de organizaciones de CJ muestran la importancia de visibilizar la historia que el producto porta. Aquí nos parece importante desarrollar — a su vez- esas alianzas de clase de las que dimos cuenta precedentemente y que puedan servir para el desarrollo de canales específicos (trabajadores y productores, estudiantes y productores), y permitiendo que estos actores se apropien de los canales de comercialización teniendo una participación activa en la gobernanza de las mismas.

\section{Conclusiones}

El CJ, como parte integrante de la agenda de promoción de la ESS, tiene una vocación transformadora de las reglas de comercio mercantiles, no sólo promoviendo canales alternativos de comercialización sino también llevando a cabo actividades de difusión y concientización de los principios que lo rigen. Ellos tienen que ver, fundamentalmente, con la creación de oportunidades para grupos desfavorecidos a través de una producción y comercialización basadas en principios éticos, donde la consideración de aspectos laborales, ambientales, sociales, culturales-identitarios y género no esté ausente. En este marco, el Proyecto de Extensión "Promoción del comercio justo como herramienta de fortalecimiento de organizaciones sociales en el Gran Santa Fe" del que los autores de este trabajo forman parte, se orienta al trabajo con grupos de productores pertenecientes al espacio rural y/o urbano-rural de Gran Santa Fe. Este tipo de espacios está atravesado por transformaciones de las que emergen "nuevas ruralidades" (nuevas prácticas y usos del territorio), que configuran nuevas iniciativas de desarrollo rural como respuesta a las tendencias concentradoras y expulsoras de la agricultura industrial.

Así, las actividades que se están llevando adelante desde el Proyecto tienen que ver principalmente con los siguientes ejes: 1) construcción de una identidad colectiva, por parte de los grupos de productores, basada en los principios del CJ;

2) adopción al interior de estos grupos de esquemas/reglas/ mecanismos de funcionamiento basados en los principios del CJ; 3) estandarización y presentación de los productos comercializados;

4) generación de vínculos con organismos e instituciones para la obtención de servicios de apoyo y financiamiento;

5) contribución a la visibilización de las organizaciones y favorecer un mayor reconocimiento del CJ y de sus principios.

Vemos con optimismo el trabajo de muchas otras organizaciones, movimientos de base, organismos, etc., que van abriendo el camino de nuevas formas de producción y circulación de la riqueza. Desde el proyecto, y como miembros de la Universidad Nacional del Litoral, aspiramos a apuntalar aquellos grupos de hombres y mujeres que se encuentran trabajando en la construcción de otra economía, dando visibilidad y reconocimiento a las actividades que a diario realizan. 


\section{Referencias bibliográficas}

Abramovich, A. y Vázquez, G. (2007). Experiencias de la Economía Social y Solidaria en la Argentina. En Revista Estudios Fronterizos, 8(15), 121-145. México: Universidad Autónoma de Baja California Mexicali.

Altieri, M.A. y Toledo, V.M. (2011). The agroecological revolution in Latin America: rescuing nature, ensuring food sovereignty and empowering peasants. In Journal of Peasant Studies, 38(3), 587-612.

Arnold, B.C. y Galán, F.S. (2014). Los mercados sociales. La economía solidaria en acción transformadora. En Documentación social, (174), 95-116.

Casadinho, J.S. (2012). L'agroécologie: bases scientifiques, histoire locale et stratégies de production (27-53). En Goulet, F. et al. (ed) L'agroécologie en Argentine et en France: Regardscroisés. París: L'Harmattan.

Cittadini, R.; Caballero, L.; Moricz, M. y Mainella, F. (2010). Economía social y agricultura familiar. INTA. Disponible en: http://inta.gob.ar/documentos/economia-social-y-agricultura-familiar/ (recuperado el 5 de abril de 2015).

Coraggio, J.L. (2004). La Economía Social como vía para otro desarrollo social. En Coraggio, J.L. De la emergencia a la estrategia: más allá del alivio de la pobreza. Buenos Aires: Espacio Editorial.

Coriat, B. y Weinstein, O. (2004). Institutions, échanges et marches. Revue d'économie industrielle, 107(1), 37-62.

Coscione, M. (2013). Agronegocios, comercio justo y cambio climático los desafíos para los pequeños productores organizados. En Otra Economía, 7(13), 133-142.

Craviotti, C. (2007). Tensiones entre una ruralidad productiva y otra residencial: El caso del partido de Exaltación de la Cruz, Buenos Aires, Argentina. En Economía, Sociedad y Territorio, VI (23), 742- 774.

Fridell, G. (2006). Comercio justo, neoliberalismo y desarrollo rural: una evaluación histórica. En ĺconos, (24), 43-57.

Gomes, R. (2007). Relaciones Norte-Sur y Sur-Sur en el Comercio Justo: Retos y Perspectivas. En Angulo, N. \& Fernández, M. (eds.), Afirmando prácticas democráticas y estrategias solidarias para un desarrollo sustentable, Memoria del II Encuentro Latinoamericano de Economía Solidaria y Comercio Justo. Lima: Grupo Red de Economía Solidaria del Perú (GRESP) y Universidad de La Habana.

IFAD-Fondo Internacional para el Desarrollo Agrícola (2009). Buenas prácticas en cartografía participativa. Disponible en: http://www.ifad.org/pub/map/pm_web_s. pdf (recuperado el 5 de abril de 2015).

King, B.G. y Pearce, N.A. (2010). The contentiousness of markets: Politics, social movements, and institutional change in markets. En Annual Review of Sociology, (36), 249-267.

Lang, T. (2010). From value-for-money' to values-for-money’? Ethical food and policy in Europe. En Environment and Planning A, (42), 1814-1832.

Lazzaretti Picolotto, E. (2008). Novos movimentos sociais econômicos: economía solidária e comércio justo. En Otra Economía, I/ (3), 74-92.
Le Velly, R. (2011). Si loin, si proches: la difficile association entre circuits courts et commerce équitable. En Revue Tiers Monde, (3), 133-149.

Luna García, A. (2006). Los espacios de la Alter-globalización: geografías y movimientos sociales (191-210). En Nogué, J. \& Romero, J. (eds.), Las otras geografías. Valencia: Tirant lo Blanch.

Mikellsen, C. (2007). Aconteceres pasados y actualidad del espacio rural pampeano argentino. Ponencia presentada en $1^{\circ}$ Congreso de Geografía de Universidades Nacionales "Pensando La Geografía En Red”, junio de 2007. Universidad nacional de Río Cuarto

Nelson, P. (1970). Information and Consumer Behaviour. En Journal of Political Economy, 78, 311-329.

Nogar, G. (2008). La multifuncionalidad territorial como escenario de la nueva ruralidad. En: PAMPA, Revista Interuniversitaria de estudios territoriales, (III), 3. Santa Fe, Argentina: Ediciones UNL.

Polanyi, K. (1989). La gran transformación. Crítica del liberalismo económico. Madrid: Ediciones de La Piqueta.

Rodet, D. (2015). L'économie solidaire comme mouvement social. Des dispositifs de qualité pour exprimer, agir et mobiliser. En Revue Française de Socio-Economie, 1(15), 195-214.

Stassart, P.M. et al. (2012). L'agroécologie: Trajectoire et potentiel pour une transition vers des systèmes alimentaires durables. In Van Dam, D. et al. (éd.), Agroéocologie, entre pratiques et sciences sociales. Dijon: Educagri éditions.

Swedberg, R. (2005). Markets in society (233-253). In Smelser, N. \& Swedberg,

R. (eds.), The handbook of economic sociology. New York: Priniceton University Press-Russell Sage Foundation.

Tetamanti, J.M y Escudero, B. (comps.) (2012). Cartografía social: investigaciones e intervención desde las ciencias sociales: métodos y experiencias de aplicación. $1^{\text {a }}$. ed. Comodoro Rivadavia: Universitaria de la Patagonia.

Valero, Y. (2013). El Comercio Justo: ¿Una herramienta para la construcción de Otra Economía? Análisis de dos experiencias de Comercio Justo en Ecuador y Argentina. Tesis de Maestría en Economía Social, Universidad Nacional General Sarmiento. Disponible en: http://www.ungs.edu.ar/ms_ungs/wp-content/uploads/2014/05/Tesis_Valero_Azuaje.pdf

Van der Ploeg, J.D.; Jingzhong, Y. y Schneider, S. (2012). Rural development through the construction of new, nested, markets: comparative perspectives from China, Brazil and the European Union. In Journal of Peasant Studies, 39 (1), 133-173.

Zibechi, R. (2010). Políticas sociales, gobiernos progresistas y movimientos antisistémicos. En Otra Economía, IV(6), 32-42.

Zusman, P.; Hevilla, C. y Molina Quijano, M. (2006). Las geografías de los tiempos lentos (255-268). En En Nogué, J. \& Romero, J. (eds.), Las otras geografías. Valencia: Tirant lo Blanch. 